\title{
Current practices for access, compensation, and prioritization in biobanks. Results from an interview study
}

\author{
Holger Langhof ${ }^{1} \cdot$ Hannes Kahrass ${ }^{1} \cdot$ Thomas Illig $^{2} \cdot$ Roland Jahns $^{3} \cdot$ Daniel Strech ${ }^{1,4}$ \\ Received: 27 February 2018 / Revised: 2 July 2018 / Accepted: 5 July 2018 / Published online: 8 August 2018 \\ (c) European Society of Human Genetics 2018
}

\begin{abstract}
Human biological materials and related data stored in biobanks are valuable resources for biomedical research. Transparent, effective, and efficient governance structures and procedures for access, compensation, and priority setting are needed, but recent debates indicate challenges in the practical application of such governance processes. This study aimed to assess the practical experiences and attitudes of biobank experts regarding the governance of biosample access, prioritization, and compensation. Qualitative, semi-structured telephone interviews were conducted with 20 biobank directors from eight countries. Respondents highlighted the need for sound governance structures in order to ensure acceptance by all stakeholders (patients/donors, researchers, research funders, public, and others). They stressed practical difficulties in trying to make best use of biomaterials. As biobanks often form part of larger academic and clinical settings, the different and sometimes conflicting interests of researchers, clinicians, patients, funders, and biobank staff currently affect the governance of access decisions. Investments such as intellectual input, financial, and human resources need to be compensated adequately. Biobanks thereby have a dual role stewarding the hosted biosamples and acting as a service provider for local researchers from universities or hospitals. In order to facilitate efficient use of human biological materials, greater harmonization of at least minimum standards for access and compensation are required at both a national and an international level.
\end{abstract}

\section{Introduction}

Human research biobanks are widely recognized as valuable resources for biomedical research [1]. The ultimate aim of biobanking is to build and run quality-controlled

Electronic supplementary material The online version of this article (https://doi.org/10.1038/s41431-018-0228-x) contains supplementary material, which is available to authorized users.

$\triangle$ Holger Langhof

Langhof.holger@mh-hannover.de

1 Institute for History, Ethics and Philosophy of Medicine, Hannover Medical School (MHH), Hannover, Germany

2 Hannover Unified Biobank, Hannover Medical School (MHH), Hannover, Germany

3 University Hospital of Wuerzburg, Interdisciplinary Bank of Biomaterials and Data Wuerzburg (ibdw), Wuerzburg, Germany

4 Charité - University Medicine Berlin, QUEST - Center for Transforming Biomedical Research, Berlin Institute of Health (BIH), Berlin, Germany storage facilities and infrastructures to enable future biomedical research [2]. Thus, access to biosamples and their associated data needs appropriate governance structures in order to best support the goal of progress in biomedical research [2-4].

\section{Access to biosamples}

Accordingly, in recent years the ownership of and access to biosamples and related data have been debated intensively $[4,5]$. Several internationally recognized guidelines and recommendations on biobank governance offer guidance on these issues [6-8]. However, most of these provisions are of general nature and need to be adapted individually by biobanks. For instance, recent empirical research revealed a substantial lack of availability of access policies in practice [9]. Moreover, with regard to access requirements, available access policies vary widely and are neither standardized nor harmonized $[9,10]$.

Added to this, the limited availability and accessibility of biosamples for researchers has been criticized [11, 12]. For instance, a US-based survey among cancer researchers 
revealed that more than two-thirds of researchers had difficulties obtaining the required biosamples [13].

\section{Compensation for efforts}

Another obstacle to biosample access claimed in the literature is limited or zero compensation in terms of recognition for the efforts and resources invested [14]. In principle, compensation is due all biobank stakeholders directly involved in the donation, collection, processing, and qualitycontrolled storage of biosamples, that is, not only the biobank staff itself, but also the contributing researchers and clinicians as well as the patients/donors.

As a means of compensation, the Bioresource Research Impact Factor (BRIF) has been proposed to measure the impact of individual biobanks in biomedical research [14, 15]. The idea behind BRIF is to allow external researchers (e.g., researchers who are not affiliated with the biobank and/or have not contributed to the specific sample collection they are interested in) to access parts of the biosample collection; therefore, the biobank receives long-term recognition for the efforts to build-up and maintain its collection(s) [15]. In order to make this recognition visible, the BRIF requires a standardized citation of an individual (or several) biobank(s) in publications using its (or their) biosamples [16]. However, the BRIF is still under development and so far not widely implemented; awareness of it is very limited even among biobank stakeholders in genetics [14]. Moreover, the BRIF is focused solely on the biobank itself or its institutional host (e.g. a university hospital) and thus fails to acknowledge other actors significantly contributing to biosample acquisition (e.g. clinicians and their contributing departments/institutes or individual researchers).

\section{Prioritization}

Finally, unlike data, many human biological materials are finite resources. While DNA is often available in relatively large amounts and DNA analysis methods are available that can rapidly analyze most of the whole genome, postgenomic research (transcriptomics, metabolomics, and proteomics) mostly uses other biological materials, such as plasma, serum, tissue, and urine. These biosamples might be valuable to a multitude of research projects, and are normally scarce as they are often fully consumed by those projects. Thus, related to the question of access decisions, another important issue to consider is how to prioritize the allocation of such relatively scarce biosamples.

\section{What is the current practice of governance?}

Several empirical studies demonstrate heterogeneous and partly insufficient application of governance procedures in practice $[9,10,17,18]$. In order to improve this situation, it is essential to have a comprehensive understanding of the currently applied spectrum of governance procedures and elements. What are the practical experiences of personnel in charge of the management and governance of human biobanks? What obstacles and challenges do they face, and how do they currently deal with them?

We aimed to assess the perceptions of biobank experts regarding the governance of "biosample access" in three areas: (1) assessing the spectrum of different approaches to access governance in practice, and challenges that might occur in this regard, (2) assessing the spectrum of procedures to compensate both biobanks and contributing clinicians/researchers for their efforts, and what kind of experiences have been made in this regard, and (3) assessing the current positions and experiences regarding situations where access is subject to priority-setting decisions.

\section{Methods}

We performed semi-structured, open-ended interviews with biobank experts, that is, persons in responsible positions at biobanks and with wide competency in the overall governance of the respective biobank (e.g., director, scientific head, Principal Investigator, or comparable). The study protocol was submitted to the Hannover Medical School Ethics Committee and was approved by a positive vote following an expedited process (EC number 3324/2016).

Based on a review of current debates reported in the literature, and drawing on our own previous studies [9], we developed an interview guide to carry out semi-structured interviews. The full interview guide is available as supplementary material (Supplementary 1).

\section{Sampling}

To identify the experts, we followed four different approaches: first, we randomly contacted seven heads of German biobanks. Second, we identified experts via the program outlines of international biobank conferences. Third, biobank registries and catalogs such as BBMRI [19], P3G [20], Eurobiobank [21], and the website Specimen Central [22] were used to identify experts and fourth, in most interviews, we asked the participant if he or she could name further potential experts. Thus, our sampling was purposive, because we aimed to assess the diversity of existing perceptions and relevant expertize.

All interviewees were sent the same invitation letter (in German for the German experts and English for the international experts) and signed a written consent form. 
Table 1 Spectrum of themes and topics regarding access, compensation, and prioritization

Main theme A: access elements

\section{General}

1.1 Development of access inquiries in the next few years

1.1.1 International cooperation of biobanks necessary

1.1.2 Access will increase

1.1.3 Access will not increase

1.2 Number and origin of access requests

1.2.1 Main origin of requests

1.2.1.1 Mostly local or national access requests

1.2.1.2 Many requests for usage in international collaborations

1.2.1.3 Number of international external requests

1.2.2 Industry access

1.2.2.1 No use of samples for commercial purpose

1.2.2.2 Usage according to overall biobank goals

1.2.2.3 Patient acceptance of industry access

1.2.2.4 Access only in cooperative projects

1.2.2.5 Access equal for industry and academia

1.2.2.6 Access for industry only for scientific projects

1.2.3 Number of access requests

1.2.4 Time needed for access procedure and decision

2. Access policy

2.1 Challenges

\subsubsection{Time-consuming}

2.1.2 Sensitive internal issues

2.1.3 Lack of personal resources

2.1.4 Technical challenges

2.2 Adapting national standards to increase local compliance

2.3 Access policy exists

2.4 Online availability

3. Access committee

3.1 Access decisions

3.1.1 Informal sample sharing

3.1.2 Using materials is important

3.1.3 Broad access

3.1.4 Researcher right to appeal

3.1.5 Scientific rationale

3.1.6 Refusal of access is not common

3.1.7 Reasons for access refusal

3.1.7.1 Refusal because access is not covered by IC

3.1.7.2 Refusal because of questionable scientific rationale

3.1.7.3 Refusal because of objection from individuals

3.1.7.4 Refusal because of ethical concerns

3.1.7.5 Refusal because of limited quantity of samples

3.1.7.6 Refusal because of overlap with local research

3.1.7.7 Refusal because of poor reputation of researchers

3.1.8 Strategies for usage of samples

3.1.8.1 Keep final aliquots
Table 1 (continued)

Main theme A: access elements

3.1.8.2 Final aliquots always for local clinic

3.1.8.3 Sample usage: turnaround of $N$ years desired

3.1.9 Challenges in access decisions

3.1.9.1 Limited information available

3.1.9.2 Huge amount of samples requested

3.1.9.3 No ethical challenges, only technical

3.1.9.4 Sometimes access process too long

3.1.9.5 Access requires consent of various actors (biobank, REC etc.)

3.2 Access inquiries and processing

3.2.1 Inquiry $\rightarrow$ REC $\rightarrow$ BB board $\rightarrow$ information to contributing researcher

3.2.2 Inquiry $\rightarrow$ REC decisions $\rightarrow$ BB board

3.2.3 REC approval for BB, no study-specific REC ap. necessary

3.2.4 BB basic service provider $\rightarrow$ not involved in decision

3.3 Internal peer review process

3.3.1 Decision is made within the research consortium

3.3.2 Decision made by scientific committee/consensus

3.3.3 Rota system: lead reviewer proposes decision

3.4 External peer review (on demand)

3.4.1 International review panel

3.4.2 Recruitment of external reviewers

4. Veto rights

4.1 Veto for internal faculty members

4.2 Reasons for veto

4.2.1 Cost-intensive samples

4.2.2 Lack of scientific knowlege gain

4.2.3 Concurrent research interests

4.2.4 Samples needed in own projects

4.2.5 Samples are scarce

5. Ownership

5.1 Shared ownership

5.2 Donor/patient

5.3 Contributing researcher

5.4 Institute/clinic

5.5 Faculty/university

6. Biobank roles: perception and challenges

6.1 Challenges

6.1.1 Dilemma faced by biobank heads

6.1.1.1 Scientific evaluation of internal projects

6.1.1.2 Conflicts of interest

6.1.1.3 Biobank needs more autonomy

6.1.2 Competition of research in globalised research environment

6.1.3 Internal projects subject to less rigorous scientific review

6.1.4 Trust building with clinicians necessary

6.2 Roles of research biobanks

6.2.1 As facilitator for open science

6.2.2 As consultant to improve research projects 
Table 1 (continued)

Main theme A: access elements

6.2.3 As institution that does research itself

6.2.4 As collector and distributor of data obtained in previous projects

6.2.5 As extra service provider (e.g. sophisticated analysis)

6.2.6 As basic service provider (primarily storage)

6.2.7 Quality safeguard (e.g. as certified test laboratory)

6.2.8 As institution that initiates cooperations (for local researchers)

6.2.9 As steward of the collection

6.3 Strategies

6.3.1 Public visibility of biobank and databases

6.3.2 Same procedures for internal and external applicants

6.3.3 Biobank as gatekeeper

6.3.4 Advantages of broad consent

6.3.5 Moratory for collections of internal researchers

Main theme B: compensation

1. Strategies

1.1 Tracking of acknowledgements

1.2 Biobank impact factor

1.3 Profit sharing

1.4 Benefit sharing for patients

1.5 Co-authorship

1.5.1 Compensation for collecting actors when material is used by others

1.5.2 Sanctioning non-compliance

1.5.3 Acknowledgement of biobank as honorarium for good work

1.5.4 No co-authorship or other compensation for clinician

1.5.5 Publications most important output

1.5.6 Always engage local researchers

1.5.7 Only acknowledgement required

1.5.8 Tracking of publications

1.5.9 Co-authorship requires scientific contribution

1.5.10 Mandatory co-authorship

2. Financial aspects of sample access

2.1 Difficulty of periodic funding

2.2 Sufficiency of funding

2.3 Baseline financing by faculty

2.4. Cost-recovery

2.4.1 Pricing according to the effort needed to store

2.4.2 Pricing in comparison to other

2.4.3 Raising of cost-recovery fees for requesting researchers

2.4.4 Access is free of charge

2.4.5 Need to increase prices

2.4.6 No increase of the fee over time

2.4.7 Charge per publication

2.4.8 Pricing depends on scarcity of material

2.4.9 Prices are subsidized

2.4.10 Prices for "older" samples cannot be fully covered
Table 1 (continued)

Main theme B: compensation

2.4.11 Biobank does not recover costs

2.4.12 No profit allowed

2.5 External versus internal access requests

2.5.1 Owner/collector of the material does not pay

2.5.2 Lower prices for local researchers

2.5.3 Prices different for external researchers

2.5.4 Competitive prices for industry

2.5.5 Prices do not discriminate internal/external

Main Theme C: Prioritization and sample values

\section{Challenge of prioritization}

1.1. Sometimes material runs short

1.2 Keeping samples driven by self-interest

1.3 In theory important

1.4 Not yet necessary

2. Strategies to avoid priority-setting decisions

2.1 Building new collections

2.2 Storing smaller units

2.3 Cooperation

2.4 Continuous sampling

2.5 Increase volume of samples

2.6 Spare access

2.7 National harmonization and coordination

2.8 Technical

2.8.1 Releasing redundant aliquots first

2.8.2 Enhance technical possibilities and methods

2.8.3 Centralized data bank for open use

3. Future perspectives

3.1 There is no need for prioritization

3.2 Prioritization is an important upcoming issue

3.3 Increasing need to prioritize desirable

3.4 Need to prioritize will increase in future

4. Criteria for prioritization

4.1 Researcher-related

4.1.1 Incentives given by requestor (e.g. gifts)

4.1.2 Chance for long-term cooperation

4.1.3 Qualification/former projects of requesting researchers

4.1.4 Financial resources of PI

4.1.5 Local researchers get priority

4.1.6 Involvement of local institution

4.1.7 Capacity (including infrastructure) of the requiring person

4.2 Sample-related

4.2.1 Projects with higher number of cases preferred

4.2.2 Rare samples allocated more generously

4.2.3 Small quantity access prioritized

4.3 Impact for society

4.4 Scientific 
Table 1 (continued)

Main Theme C: Prioritization and sample values

4.4.1 Prospect of high-ranked publication

4.4.2 Innovative study design

4.4.3 Quality of proposal

4.4.4 Scientific value

4.4.5 General promise of proposal

4.4.6 Objectives

4.4.7 Diagnostic testing has priority

4.5 Rotation of approvals

4.6 First-come-first-serve

4.7 Accordance with biobank charter goals (e.g., disease-specific)

4.8 Promise to share data

5. Sample value

5.1 Selective collection of valuable samples

5.2 Sample itself has no value, usage is key

5.3 The more data connected, the more scarce are samples

5.4 Likelihood of usage

5.5. Pharma research and development

5.6 Rare samples higher value

5.7 Follow-up makes samples more valuable

5.8 Broad access is important

5.9 Sample value increases over time

5.10 Certain amount of samples should not be used for the first few years

5.11 Special treatment in the follow-up period

5.12 Indications that are requested more frequently

5.13 Sample was taken a short time before disease appearance

5.14 Linked data and samples more valuable for research

\section{Analysis}

Thematic text analysis to extract, analyze, and synthesize all relevant information was applied to all interview transcripts [23]. The first 13 transcripts were analyzed by one author alone (HL). Relevant interview passages were identified inductively, and a descriptive code was applied. Text passages and codes were matched in order to collate and build thematic clusters. Following this approach, the findings were clustered into a matrix of thematic topics and subtopics.

This matrix was checked for validity and consistency by another researcher (HK). Through extensive discussion within the research group, the matrix was further refined in order to best synthesize the information. Two researchers (HL, HK) then analyzed the remaining seven transcripts independently, using the refined matrix. Analysis was complemented by adding and further modifying codes until preliminary thematic and conceptual saturation was achieved for the main topics and first-order subtopics. Thematic and conceptual saturation implies that no new topics (themes or broader concepts) can be generated to extend the matrix. This resulted in a matrix of broad themes and narrow subthemes and topics.

One other researcher (DS) then checked all transcripts and the resulting matrix. In a one-day workshop, the findings were discussed and slightly modified to allow for internal consistency and external validity. A final matrix was agreed by all researchers engaged in the analysis (HL, HK, and DS).

All qualitative analysis was performed using MaxQDA [24].

\section{Results}

Of 37 biobank experts contacted, 20 agreed to participate. Of the 20 interviewees, eight were from Germany, four from the United Kingdom, two from the Netherlands, two from Sweden, and one each from Luxembourg, Switzerland, Israel, and South Africa. All interviewees worked in academic research settings, e.g., biobanks based at university hospitals.

The key result (primary outcome) of this interview study is the qualitative spectrum of themes and topics that biobank experts mentioned when interviewed about current decisionmaking regarding the three overarching themes: (1) biosample access, (2) compensation procedures, and (3) perception of/experience with prioritization. Table 1 specifies the three overarching themes and presents the themes with their specific topics in more detail (see also supplementary table 2 for exemplary quotes for each specified theme and respective topics). In the following, we present a brief selection of topics and quotes in an illustrative way to facilitate the understanding of the qualitative spectrum.

\section{Biosample access}

Asked for their practical experiences with deciding on sample access, the respondents provided a broad range of distinctive elements that frame their access decisions. These elements are (1) the adoption of an access policy, (2) the access committee, (3) veto rights and the appointment of gatekeepers, (4) ownership questions, and (5) the role of biobank in sample access governance.

\section{Access committees}

An important element of sample access is the appointment of a scientific board to review access inquiries and project proposals. The respondents reported significant variation in the type and scope of these boards. Besides different names for the board (e.g., scientific review board, management committee, etc.), they also varied in terms of their working structure. For instance, while in some boards decisions are made only by majority vote, one 
respondent highlighted the possibility of a "rota system" with one defined lead reviewer:

“(..) we got pathologists, surgeons, molecular scientists, the whole sort of range of people. [...]But that was taking too long. So what we have actually done now is there will be lead reviewer [...]. So whoever comes up to the top of the list next, they will review the application. They will let the rest of the panel know what their decision is and only if somebody disagrees with that decision will it then go out for a wider review to the whole panel." I7

Virtually all respondents highlighted the need for a sound review system in order to ensure scientific quality and the best use of the stored resources:

“(...) you have to think about the ethics of access as well. And so we've taken the standpoint that material that you take from a patient where you've consented and said it's going to be used in research you almost have a contract with that patient to make sure that really it's used in research. So you should make it as widely available as possible but it has to be good science otherwise it's a misuse of the sample and actually you've broken the contract with the patient." I12

\section{Veto rights: the role of gatekeepers}

Another important access element is the appointment of gatekeepers with a veto over local clinicians or researchers, in order to maintain a degree of control over the biosamples. One particular reason why biobanks allow such veto rights is competition. For instance, one respondent highlighted:

"So the PI can say, sure, I will accept a request to view the samples from a researcher who is working in a different field. But if it's like a breast cancer study, if it's his biggest competitor who wants to access those samples then he or she might say, no, I am still using them for that purpose, I won't allow it. And that seems reasonable to me." I8

\section{Different ownership models}

The respondents also mentioned differing ways in which ownership is dealt with in practice. While acknowledging the particular legal provisions that may vary between countries, most respondents highlighted that a core challenge with regard to ownership is the role of local scientists who contribute to the biobank by collecting the biosamples:

"The legal aspect is one thing. Then the other thing is the PI (...). They feel a strong sense of ownership (...) and they view the samples as theirs." I9

One currently applied strategy on how to deal with this challenge is an access model with "shared ownership":

"Okay so in general the way we function is half of the aliquots belong to the PI okay so then we do all the work, all the service to constitute his collection for his research, so he's very happy with that and the other half of the collection is ours, the custodianship of the biobank." I11

See Table 1 for further ownership models that were mentioned in the interviews.

\section{Procedures for compensation}

In general, all biobank experts agreed that the question of compensation is of utmost importance in practice. Here again, the respondents expressed a range of procedures for how compensation is governed. The procedures range from a "mandatory co-authorship" to "only acknowledgments" and from access "free of charge" to "charge per publication". Generally, the question of compensation is dominated by some sort of academic recognition rather than monetary compensation. For local researchers, who contributed to the collection, the commonest sort of compensation is the prospect of coauthorship. One respondent stated very clearly that:

"They are all scientists and they say: yes, I will share the samples, but I want something back, not money, but I want to be at least acknowledged in the publication or listed as co-author" I2 (translated by the authors)

However, it was stressed that co-authorship must not be promised gratis, but subject to clear provisions, and requires some intellectual contribution to the specific research project: Moreover, one respondent also highlighted the need for a good sense of proportionality, especially when collections have evolved over a long time:

"We try not to exaggerate that. (...) well there are two things: We encourage that always a local researcher 
could be involved in the project, which could make sense in many ways. First of all it makes sense, because then it is easier for the external research group to find their way around to have an internal expert. (...) And they (...) are almost in every case very happy for that. (...) if this person contributes scientifically, we would say that he should or she should probably [be] offered a co-authorship. But only if it is played by the banker rules. Sometimes when people ask for request to let's say diabetes data, where there is a diabetes research group with a PI that has been 20 years of his life to organize this, followup and prepare data, then it is obvious that he would be offered a co-authorship." I8

For biobanks to be compensated, the Bioresource Research Impact Factor (BRIF) was seen as a valuable instrument, but respondents also highlighted that it will still take some time for it to fulfill its potential, mainly because it is not yet popular enough.

In some cases, even the (voluntary) donors are indirectly involved in the governance of compensation. For instance, one respondent stressed the possibility for industry stakeholders to access biosamples, when in return they agree to provide the projected product to the donors:

"I don't want to be negative with academic research but in general the output of academic research is a paper publication. So then from the outcome of academic research we can only argue about the general benefit for the future generations. (...) if it's a request coming from industry then there is much more chance that there is a practical application, either a diagnostic kit or pharmaceutical product or, you know, a new method that is validated and that would be applied and in the real everyday life and there can be a direct benefit, practical benefit to the population. So in this case we have tried (...) a clause where the local hospital from which the patients have been recruited, the samples have been collected that there is for example a limited license maybe for a certain period of time, for the use of the product, the diagnostic kit or pharmaceutical (...)" I11

Please refer to Table 1 for further categories and specifications that fall under the overarching theme of "compensation".

\section{Perceptions of and practical experiences with priority setting}

For most respondents, prioritization of access was only occasionally an issue. However, most respondents agreed that this might become a challenge in future. Indeed, some argued that they would appreciate being faced by prioritysetting challenges as this could serve as an indicator for a popular and well-appreciated (high quality) biobank:

"So, even though (...) more customers will probably mean more challenges in terms of prioritizing, it is a matter of, I would say, life and death for a biobank to have customers. " I8

When asked how they handle situations in which they need to prioritize - even if only theoretically, because it has not yet been necessary for them-the respondents mentioned plenty of decision criteria (please refer to Table 1 for details). These criteria broadly divide into criteria related to (1) requesting researchers (e.g. degree of qualification), (2) scientific merits (e.g. innovative study design), and (3) biosample-related criteria (e.g., projects requiring small amounts of samples are prioritized).

The value of different biosamples was another topic that arose in the context of priority-setting discussions. Here again, respondents provided a wide range of viewpoints. While many stated that the value of biosamples is determined by different variables (e.g., rarity, quantity, and quality of related data, etc.), others argued that a priori all biosamples are of same value, but that it is the concrete use/ analysis that makes it valuable. Please refer to Table 1 for further categories and specifications that fall under the overarching theme of "prioritization".

\section{Discussion}

In this in-depth interview study, we assessed the qualitative spectrum of experiences regarding the governance of biosample access, prioritization, and compensation in the practice of 20 international research biobank experts. In the following, we discuss the results against the background of currently existing regulations and policies, and will give an outlook on future developments.

\section{Heterogeneity and lack of harmonization}

One key finding that applies to all of the three governance topics access, compensation, and prioritization is the multitude of different approaches in practice. While this might to some extent rely on national or regional particularities (e.g., data protection rules) or on different (research) core areas of the biobanks (e.g., rare diseases versus common diseases), this finding could also imply potential barriers for the overall goal of an international research framework that aims to facilitate rapid access to human biological materials 
provided by different biobanks. In Europe, for instance, BBMRI-ERIC was established in order to develop and operate a pan-European distributed research infrastructure of biobanks and biomolecular resources with the overall goal of facilitating access to resources.

\section{Interest in harmonized policies for access and compensation}

Despite the current differences in how access and compensation is governed, all interview participants indicated a general interest in having more standards and harmonization, as this will support the overarching goals of networked biobank research. Therefore, future policy development for access and compensation in biobank research should explicitly acknowledge the status quo as demonstrated in this paper and propose minimum standards necessary to facilitate networked biobank research. The same policies and guidelines should also indicate examples for how individual biobanks can acknowledge local particularities in their governance framework without compromising their contribution to networked biobank research.

\section{Balancing of interests}

Most of the respondents in our interview study were highly supportive of research using the biosamples stored in their facilities and emphasized both the importance of international networking and sharing of resources, and their willingness to contribute to this goal. At the same time, the respondents virtually all agreed that a biobank needs local researchers/clinicians to collect and contribute biosamples and that a biobank is therefore necessarily embedded in a larger local research setting and its particular interests. Furthermore, biobank governance has to acknowledge that the interests of patients (who donate their biosamples and related health data) and the interest of the public (via public funding organizations and tax-based support of universities) are substantial contributors to the biobank infrastructure.

It was stressed that local researchers/clinicians, often physicians, often seem to consider the biosamples as their own; although, the question of (legal) ownership is still controversial [25-27]. While the interview participants questioned physicians' perceived "right" to control the use of locally stored biosamples that they collected as part of their publicly funded job profile, they found it obviously legitimate for this group of researchers to be appropriately compensated for their contributions to the biobank.

As most biobanks are publicly funded, partly by direct grants and partly by public support of their respective universities [28], biobanks should foster the use of biosamples and data for biomedical research projects that are of high scientific and/or social value [5]. Furthermore, patients/ donors (representing part of the "public") face certain risks when consenting to biobank-based research [27]. To compensate for these risks, biobank-based research should again ensure that the supported research projects promise high scientific and/or social value [29].

Against the background of the above-outlined interests in biobank-based research, biobanks do not only provide the infrastructure for the processing and quality-controlled storage of biosamples and related data but, in addition, act as stewards of the hosted resources [3, 30-33]. Many interview participants highlighted that biobanks are charged with ensuring the ethically and legally sound utilization of human biological materials and related data in addition to capturing their scientific value.

These different stakeholder interests can conflict, especially when access to biosamples for high-quality external research projects is hindered or even completely blocked. The likelihood that both social and scientific value are best promoted increases when access to biosamples and related data are generally possible for all researchers worldwide [34]. Furthermore, some innovative research questions might depend on high sample numbers and, thus, international networking might be even more fruitful. In conclusion, both innovative research projects and international collaborations can contribute significantly to the societal and scientific value of biomedical research making use of high quality human biomaterials and related data stored in quality-controlled (certified) biobanks.

Future policy development in biomedical research should, therefore, concentrate on how to balance the different interests of patients/donors, (public) funding agencies, clinicians/ researchers collecting, and biobank staff processing and storing human biological materials and, thus, acting as stewards of the hosted biosamples. In analogy with and response to the normative principle of "broad consent" given by patients/donors, clinical biobanks processing and hosting human biosamples could be guided by an overarching normative principle, such as "broad access". Such a broad access to stored biosamples might lead to competition among the best and most promising biomedical research ideas and proposals and, thus, augment the likelihood that the scientific and societal value of biomedical research will increase. However, this ideally formulated normative concept of "broad access" needs to be further developed, always respecting the historically developed local research environment as well as current political and economic factors.

\section{Prioritization}

While the issue of priority setting might not be of the utmost importance in the current practice of biobank-based research, it might soon become urgent if broader access policies are promoted and implemented. Guidelines that aim 
to introduce priority setting in future access decisions will need to determine and to define what classifies (qualifies) low versus high-value biosamples and low versus high quality biomedical research projects.

\section{Conclusion}

In order to facilitate and achieve optimum use of human biological materials in biobank-based research, further development of guidance is needed focusing on appropriate generic models for access, compensation, and priority setting, including means and strategies for conflict resolution between the different stakeholders involved in biobank-based biomedical research. The qualitative results of the present study may serve as a comprehensive source for future discussions and decision-making on such guidance for clinicians/researchers, regulatory bodies, (public) funding organizations, patient organizations, and the general public. In the future, biobanks will increasingly develop toward digital databanks that run inhouse analysis on the samples, sharing only the data without handing out larger quantities of samples. The UK Biobank is a prominent pioneer in this regard. This development might in the long run also influence questions on access to samples and prioritization.

Author contributions Conceived and designed the research study: $\mathrm{H}$. L., H.K., and D.S. Conducted the interviews: H.L., H.K. Analyzed the data: H.L., H.K. Wrote the manuscript: H.L., D.S., R.J., and T.I.

\section{Compliance with ethical standards}

Conflict of interest The authors declare that they have no conflict of interest.

\section{References}

1. Riegman PH, Morente MM, Betsou F, de Blasio P, Geary P. Marble arch international working group on biobanking for biomedical R: biobanking for better healthcare. Mol Oncol. 2008;2:213-22.

2. Mabile L, Dalgleish R, Thorisson GA, et al. Quantifying the use of bioresources for promoting their sharing in scientific research. Gigascience. 2013;2:7.

3. Yassin R, Lockhart N, Gonzalez del Riego M, et al. Custodianship as an ethical framework for biospecimen-based research. Cancer Epidemiol Biomark Prev. 2010;19:1012-5.

4. Mascalzoni D, Dove ES, Rubinstein Y, et al. International Charter of principles for sharing bio-specimens and data. Eur $\mathrm{J}$ Hum Genet. 2015;23:721-8.

5. Fortin S, Pathmasiri S, Grintuch R, Deschenes M. 'Access arrangements' for biobanks: a fine line between facilitating and hindering collaboration. Public Health Genom. 2011;14:104-14.

6. Council of Europe: Recommendation $\mathrm{CM} / \operatorname{Rec}(2016) 6$ of the Committee of Ministers to member States on research on biological materials of human origin, 2016, 2016.

7. International Society for Biological and Environmental Reposotories (ISBER. 2012 best practices for repositories collection, storage, retrieval, and distribution of biological materials for research international society for biological and environmental repositories. Biopreserv Biobank. 2012;10:79-161.

8. National Cancer Institute. NCI Best Practices for Biospecimen Resources. Bethesda, Maryland: National Institutes of Health (NIH); 2011.

9. Langhof H, Kahrass H, Sievers S, Strech D. Access policies in biobank research: what criteria do they include and how publicly available are they? A cross-sectional study. Eur J Hum Genet. 2017;25:293-300.

10. Verlinden M, Nys H, Ectors N, Huys I. Access to biobanks: harmonization across biobank initiatives. Biopreserv Biobank. 2014;12:415-22.

11. McIntyre P. Unlocking progress: why we need to change the culture of biobanking. Cancer World. 2016;72:46-50.

12. Horn EJ, Terry SF. Biobanking Challenges and Informatics Opportunities. In: Richesson RL, Andrews JE, (eds). Clinical Research Informatics.. London: Springer London; 2012. p. 221-31.

13. Massett HA, Atkinson NL, Weber D, et al. Assessing the need for a Standardized Cancer HUman Biobank (caHUB): findings from a National Survey with Cancer Researchers. JNCI Monogr. 2011;2011:8-15.

14. Howard HC, Mascalzoni D, Mabile L, Houeland G, Rial-Sebbag E, Cambon-Thomsen A. How to responsibly acknowledge research work in the era of big data and biobanks: ethical aspects of the Bioresource Research Impact Factor (BRIF). Journal of Community Genetics 2017;9:169-176.

15. Cambon-Thomsen A. Assessing the impact of biobanks. Nat Genet. 2003;34:25-26.

16. Bravo E, Calzolari A, De Castro P, et al. Developing a guideline to standardize the citation of bioresources in journal articles (CoBRA). BMC Med. 2015;13:33.

17. Pereira S. Motivations and barriers to sharing biological samples: a case study. J Pers Med. 2013;3:102-10.

18. Colledge F, Elger B. Getting a fair share: attitudes and perceptions of biobank stakeholders concerning the fairness of sample sharing. Bioethics. 2015;29:424-30.

19. Holub P, Swertz M, Reihs R, van Enckevort D, Müller H, Litton J-E. BBMRI-ERIC Directory: 515 Biobanks with Over 60 million biological samples. Biopreserv Biobank. 2016;14:559-62.

20. Ouellette $S$, Tassé AM. $P(3) G-10$ years of toolbuilding: from the population biobank to the clinic. Appl \& Transl Genom. 2014;3:36-40.

21. Mora M, Angelini C, Bignami F, et al. The EuroBioBank Network: 10 years of hands-on experience of collaborative, transnational biobanking for rare diseases. Eur J Hum Genet. 2015;23:1116-23.

22. Specimen Central: Global Biobank Directory, Tissue Banks and Biorepositories, 2017.

23. Dixon-Woods M, Agarwal S, Jones D, Young B, Sutton A. Synthesising qualitative and quantitative evidence: a review of possible methods. J Health Serv Res Policy. 2005;10:45-53.

24. VERBI Software GmbH: MAXQDA, Software for qualitative data anaylsis. Berlin, Germany: VERBI Software Consult Sozialforschung $\mathrm{GmbH}$.

25. Chalmers D. (2011) Genetic Research and Biobanks. In: Dillner J. (eds) Methods in Biobanking. Methods in Molecular Biology (Methods and Protocols), vol 675. Humana Press, Totowa, NJ, p. $1-37$.

26. Cadigan RJ, Easter MM, Dobson AW, et al. "That's a good question": university researchers' views on ownership and retention of human genetic specimens. Genet Med. 2011; 13:569-75

27. Budimir D, Polašek $\mathrm{O}$, Marušić A, et al. Ethical aspects of human biobanks: a systematic review. Croat Med J. 2011;52:262-79. 
28. Henderson GE, Cadigan RJ, Edwards TP, et al. Characterizing biobank organizations in the US: results from a national survey. Genome Med. 2013;5:3.

29. Knoppers BM, Harris JR, Tasse AM, et al. Towards a data sharing code of conduct for international genomic research. Genome Med. 2011;3:46.

30. Fullerton SM, Anderson NR, Guzauskas G, Freeman D, FryerEdwards K. Meeting the governance challenges of next-generation biorepository research. Sci Transl Med. 2010;2:15cm13-15cm13.
31. O’Brien SJ. Stewardship of human biospecimens, DNA, Genotype, and Clinical Data in the GWAS Era. Annu Rev Genom Hum Genet. 2009;10:193-209.

32. Henderson GE, Edwards TP, Cadigan RJ, et al. Stewardship Practices of U.S. Biobanks. Sci Transl Med. 2013;5:215cm217-215cm217.

33. Vaught J, Lockhart NC. The evolution of biobanking best practices. Clin Chim Acta. 2012;413:1569-75.

34. Bobrow M. Balancing privacy with public benefit. Nature. 2013;500:123. 